\title{
On-site BIPM comparison of quantum Hall effect resistance standards and their scaling to other values
}

\author{
Benjamin Rolland ${ }^{1, *}$, Pierre Gournay ${ }^{1}$, and Nick Fletcher $^{1}$ \\ ${ }^{1}$ Bureau International des Poids et Mesures, 92310 Sèvres, France
}

\begin{abstract}
This paper reviews the implementation of an on-site comparison of quantum Hall resistance standards and their scaling to other values. This comparison, organized by the Bureau International des Poids et Mesures (BIPM), is registered as BIPM.EM-K12 and has for objective the verification of the international equivalence of the representation of the ohm between National Metrology Institutes within 1 to few parts in $10^{9}$. The required equipment, some technical aspects and available results are presented.
\end{abstract}

\section{Introduction}

The practical realization of electrical units is today based, in most National Metrology Institutes (NMIs), on two quantum effects: the Josephson effect and the quantum Hall effect (QHE). The first allows to relate the volt to two fundamental constants, the Planck constant (h) and the elementary charge (e) through the Josephson constant:

$$
K_{\mathrm{J}}=\left(\frac{2 e}{h}\right)
$$

(see [1] for details), and the second effect, which is the topic of this paper, relates the ohm to the same fundamental constants through the von Klitzing constant:

$$
R_{\mathrm{K}}=\left(\frac{h}{e^{2}}\right)
$$

The QHE is a quantum version of the classical Hall effect, generated in a two dimensional electron gas (2DEG), most often realized in GaAs semiconductor heterostructures [2]. If this $2 \mathrm{DEG}$ is kept at low temperature (a few kelvin) and is subjected to a high magnetic field (flux density $B$ of the order of 10 tesla), when an electrical current $I$ is passing through the Hall bar (provided this current remains below a structural breakdown value), a Hall voltage $U_{\text {Hall }}$ appears in the transverse direction. The Hall resistance is defined as $R_{\mathrm{H}}=U_{\text {Hall }} / I$. The particularity of this resistance is that its variations versus $B$ consist in a succession of flat steps, commonly named plateaux, corresponding to flux density ranges on which the resistance $R_{\mathrm{H}}$ remains constant, with a quantized value equal to:

$$
R_{\mathrm{H}}(n)=\frac{R_{\mathrm{K}}}{n}
$$

where $n$ is an integer quantum number corresponding to the number of the plateau as shown in Fig. 1. The resistance $R_{\mathrm{H}}(n)$ is often referred to as the quantum Hall resistance (QHR) of the $n^{\text {th }}$ plateau.

In practice, NMIs implement the QHR to realize and maintain the ohm almost systematically on plateau $n=2$ using the conventional value of the von Klitzing constant, fixed in 1990 to be exactly $R_{\mathrm{K}-90}=25812.807 \Omega$.
Although the QHR can be reproduced at anytime and anywhere with an extremely low relative uncertainty of the order of 1 part in $10^{9}$ or below, the relative uncertainty of the practical representation of the ohm using the conventional value $R_{\mathrm{K}-90}$ of the von Klitzing constant is 1 part in $10^{7}$ in the International System of Units (SI), thus limiting the interest of implementing a QHR. To overcome this issue and benefit from the great potential of the QHE, a conventional unit of resistance, the $\Omega_{90}$, has been established (although the index " 90 " is in general omitted). It simply corresponds to the ohm realized from a QHR using the $R_{\mathrm{K}-90}$ value. Practically, this means that a resistance value expressed in terms of $\Omega_{90}$ could have a very low relative uncertainty of the order of $1 \times 10^{9}$ (if calibrated against a QHR) but will have an additional relative uncertainty of $1 \times 10^{7}$ if expressed in SI ohm unit. More details about the 1990 electrical units can be found in [3].

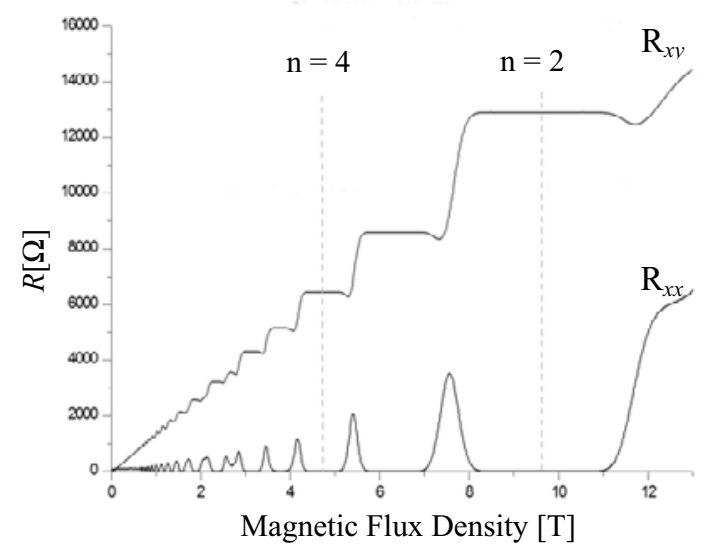

Fig. 1. Experimental signature of the Quantum Hall effect. Every plateau in the transverse resistance $R x y$ comes with a null longitudinal resistance $R x x$.

The $\Omega_{90}$ is universally used by NMIs for the dissemination of the resistance unit at dc using a QHR of value $R_{\mathrm{H}}(2)=12906.4035 \Omega_{90}$ (i.e. $12906.4035(13) \Omega_{\mathrm{SI}}$ ).

* Corresponding author: brolland@bipm.org

(C) The Authors, published by EDP Sciences. This is an open access article distributed under the terms of the Creative Commons Attribution License 4.0 (http://creativecommons.org/licenses/by/4.0/). 
It is implemented in combination with a resistance comparison bridge (as for instance the one described in [4]) allowing to calibrate a classical standard resistor against $R_{\mathrm{H}}(2)$. This resistor can be used in turn to calibrate other resistors of the same or different values by using the same comparison bridge or another scaling device.

In order to verify the international equivalence of the representation of the ohm by NMIs using a QHR, the Bureau International des Poids et Mesures (BIPM [5]) has implemented a programme of international comparisons referred to as BIPM.EM-K12. It allows NMIs to validate their implementations of the QHE for dc-resistance traceability as well as their scaling devices by comparison to the reference maintained at the BIPM.

\section{Key comparison BIPM.EM-K12}

\subsection{History}

Prior to the development of the QHE and its use for the representation of the ohm, the equivalence of the NMIs was verified through round-robin comparisons consisting of comparing measurements of classical standard resistors (generally wired-wound type) traveling between the participating NMIs and the BIPM. However, this method has a limited accuracy mainly due to unpredictable changes during the transportation related to mechanical and/or thermal shocks. Even for high quality $1 \Omega$ and $10 \mathrm{k} \Omega$ standards, the limit is a few parts in $10^{8}$ relative uncertainty [6].

From the 1990's, the growing number of NMIs implementing a QHR has offered the opportunity to compare not only classical resistance standards but QHE standards. This has led to a significant reduction of the above-mentioned uncertainty by a factor 10 . In effect, as mentioned earlier, a QHR can, in principle, be implemented anywhere at any time with always the same quantized value. For this reason, the BIPM developed a transportable QHE reference standard and the associated measurement system (Recommendation 2, CI-1988 of the Comité International des Poids et Mesures [7]) that can be transported directly to NMIs to validate on-site their traceability chain, including a quantized Hall resistance standard. This comparison is registered as BIPM.EM-K12 [8] and can be carried out with a relative standard uncertainty of about 1 part in $10^{9}$.

A first set of five comparisons were previously carried out in the 1990's, first with the BNM/LCIE (now LNE, France, 1993) then with the METAS (Switzerland, 1994), the Physikalisch-Technische Bundesanstalt (PTB, Germany, 1995), the National Physical Laboratory (NPL, United Kingdom, 1997) and the National Institute of Standards and Technology (NIST, USA, 1999). The results of these comparisons are available at [8]. At that time, no more NMIs were capable of reaching an uncertainty at a level low enough for justifying additional on-site QHR comparisons.
In recent years the importance of on-site comparisons has been recognized at several occasions because of the development of worldwide metrology, progress in measurements bridges (commercial or built by NMIs) and the large number of laboratories linking their resistance standards to the QHE to reduce their uncertainties. In the light of this need, a survey was sent to the member NMIs of the Consultative Committee for Electricity and Magnetism (CCEM) to investigate their interest in resuming the on-site QHR comparison. Fifteen laboratories have expressed the wish to participate in the coming years. The first new comparison was carried out in 2013 at the PTB [9].

\subsection{Protocol of BIPM.EM-K12 comparison}

The objective of the comparison is to realize the ohm from a QHE reference at values of $100 \Omega, 10 \mathrm{k} \Omega$ and $1 \Omega$. The comparison is not of quantum Hall devices, but of the values assigned to conventional standards of these values, derived from a QHE reference. It thus covers not just the QHE system at the NMI, but also the ratio bridge (or other scaling device) used to calibrate standard resistors.

The protocol for these on-site comparisons includes three sets of measurements (Fig. 2). First, the calibration of a $100 \Omega$ standard resistor against the QHE reference (using at present the conventional value for the von Klitzing constant, $R_{\mathrm{K}-90}$ ), with ideally a current of $5 \mathrm{~mA}$ in the $100 \Omega$ standard $(\approx 40 \mu \mathrm{A}$ through the QHR for a ratio of $1: 129)$ corresponding to a power dissipation of about $2.5 \mathrm{~mW}$ in this standard. This configuration has been chosen as it constitutes a compromise between the various limitations of breakdown current in the QHE device, signal to noise ratio and self-heating effects in the $100 \Omega$ resistor.

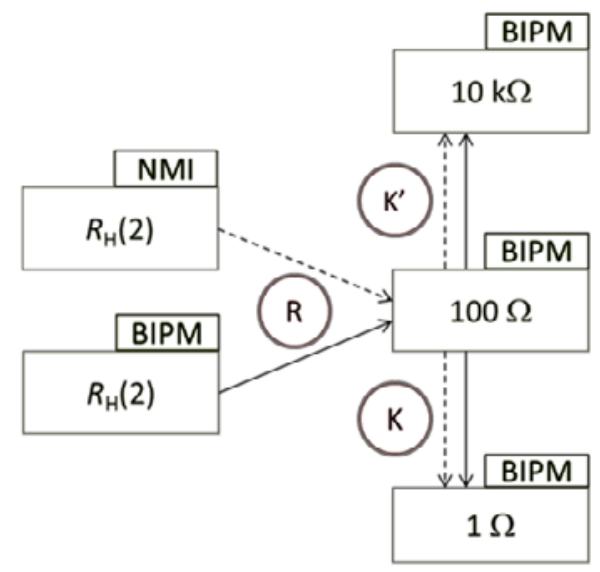

Fig. 2. Schematic representation of the measurements carried out during the comparison. Rectangles: resistances to be compared, circles: resistance $R$ and ratios $K$ to be determined, solid and dashed arrows: measurements with the BIPM's $1 \mathrm{~Hz}$ bridge and with NMI's bridge, respectively.

Subsequently the ratios $100 \Omega / 10 \mathrm{k} \Omega$ and $100 \Omega / 1 \Omega$ are realized to check the scaling device of the participating NMI. In order to minimize the effects of short term drift in the standards under test, repeated 
interleaved series of measurements are carried out. The measurands to be compared are the $100 \Omega$ dc value in term of $R_{\mathrm{K}-90}$ and the two dc ratios $10 \mathrm{k} \Omega / 100 \Omega$ and $100 \Omega / 1 \Omega$.

These measurements are performed on-site, at the participating NMI by both the BIPM, using its travelling QHE system, and the NMI, using its own QHE system. Differences are then calculated to deduce the degrees of equivalence between the NMI and the BIPM. Through the common participation of the BIPM, degrees of equivalence between different NMIs can also be obtained.

The resistance is assumed to be measured at dc, although in practice the measurement system needs to use polarity reversals to eliminate offsets and drifts. Moreover, the transportable BIPM bridge operates at $1 \mathrm{~Hz}$ (sinusoidal current) but the reference for the comparison remains the BIPM Cryogenic Current Comparator (CCC) bridge, which operates with much slower current reversals (Fig. 3). The link between the $\mathrm{CCC}$ and the $1 \mathrm{~Hz}$ bridge is made at least twice a year and the frequency coefficients of standards resistors are verified at this occasion.

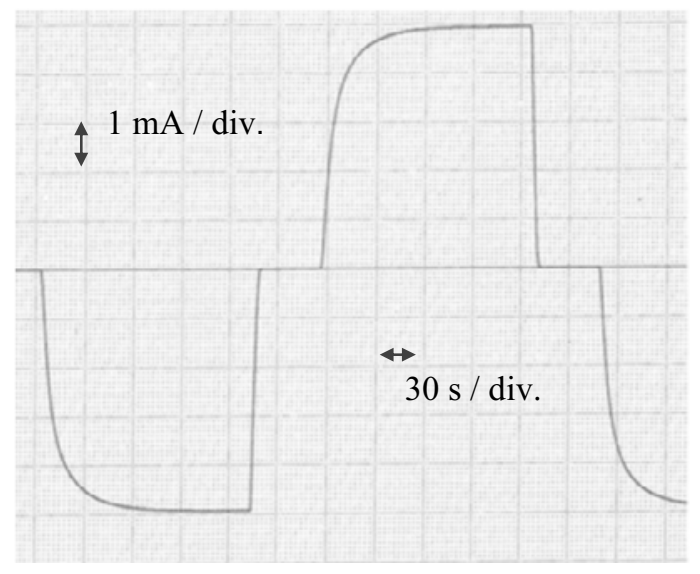

Fig. 3. Screen shot of the current applied to the resistance on the BIPM's CCC bridge and used as a reference. The amplitude is $5 \mathrm{~mA}$ (primary side) and the complete sequence is 340 seconds.

\subsection{The BIPM measurement system}

The BIPM transportable QHE reference system includes a cryostat containing a superconducting magnet and a low-temperature insert, a set of QHE samples mounted on a cryogenic probe and a room-temperature ac resistance ratio bridge operating at $1 \mathrm{~Hz}$. The bridge is equipped with two room-temperature current comparators of nominal ratios 2065/16 and 1500/15 for ratios 129.06 and 100 respectively. The current comparators comprise a single magnetic core of high permeability, wound with a tuned detection winding enclosed in electrostatic and magnetic shields and two ratio windings (highest turns number winding in series with the primary arm of the bridge and lowest turns in series with the secondary arm) using a coaxial guarding technique to reduce the relative ratio error from the typical value of $10^{-7}$ of a room temperature comparator to a $10^{-9}$ level. The principle of the double current source and the insulation requirements of the $1 \mathrm{~Hz}$ bridge (Fig. 4) is the same as in the CCC bridge. This bridge was developed and built at the BIPM and is currently used for routine measurements [10]. A double current source delivers currents $i_{\mathrm{p}}$ and $i_{\mathrm{s}}$, the ratio of which, $i_{\mathrm{p}} / i_{\mathrm{s}}$, is adjusted to be within 1 part in $10^{6}$ of the resistance ratio to be measured $R_{\text {secondary }} / R_{\text {primary }}$. A feedback loop locked on zero-voltage detection across the detection winding of the current comparator ensures the fine regulation of the current ratio. Using a loop gain of the order $10^{3}$, able to maintain stable operation during the measurements, the closed-loop error of the system is lower than one part in $10^{9}$. A detailed description of this ac bridge can be found in [11].

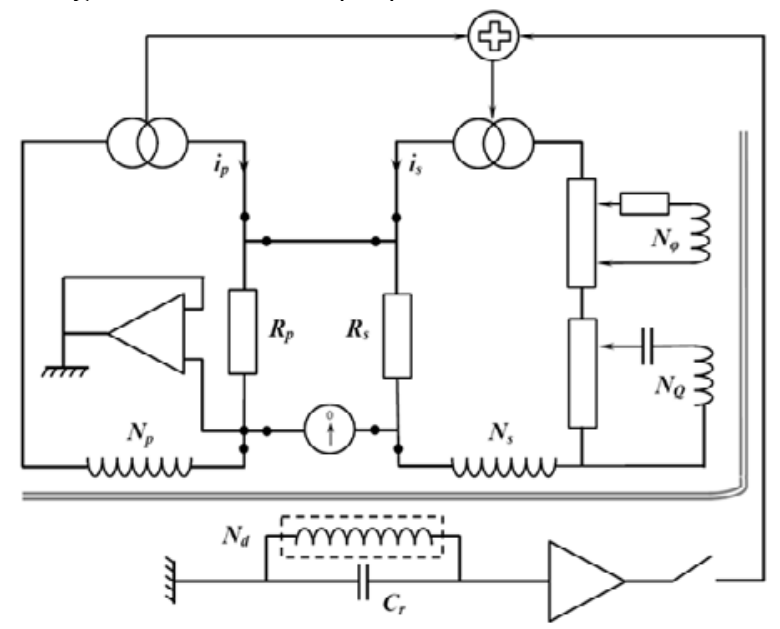

Fig. 4. Schematic of the BIPM $1 \mathrm{~Hz}$ bridge based on an ac current comparator.

\subsubsection{Advantages of using an ac system}

Using a low-frequency comparison bridge on-site is preferable to transporting the BIPM CCC bridge itself since the ac bridge has the advantage of being a more rugged instrument, simple to operate, and much less sensitive to $1 / f$ noise and thermal EMF's. Furthermore, it provides resolution and reproducibility that are comparable to those of the BIPM CCC [12].

The ac bridge currently used is an improved copy of the one used for the first series of comparisons. All elements are mounted in flight cases and the setup can be installed in a couple of hours (Fig. 5).

\subsubsection{The standard resistors}

Three transfer resistance standards are used during the comparison. They are highly stable commercial resistance standards of $1 \Omega, 100 \Omega$ and $10 \mathrm{k} \Omega$, installed in home-made temperature-controlled individual enclosures working at $25^{\circ} \mathrm{C}$ with regulation accuracy as low as few $\mathrm{mK}$ under "normal" laboratory conditions. They are used as 5 terminal resistors with two high and low current terminals and two high and low voltage 
terminals for measurement, and a case connection also used as a shield.

Temperature control is made using a Peltier cooling module in parallel with resistive heaters. An analog PID controller was specifically developed to avoid any clock or pulse width modulation signals and harmonics that could disturb the measurement bridges during the comparison.

For these conventional standards, the differences between values measured at $1 \mathrm{~Hz}$ and at dc are small, but not negligible. As mentioned above, these differences are determined at the BIPM prior to the comparison by comparing measurements performed at $1 \mathrm{~Hz}$ with the transportable bridge and at slowly reversed dc with the CCC bridge. They are applied as corrections to the measurements carried out at $1 \mathrm{~Hz}$, meaning that the ac bridge is used as a transfer instrument referenced to the BIPM CCC. Corrections are of few parts in $10^{9}$ for the $10 \mathrm{k} \Omega$ and $100 \Omega$ standards. They are higher for the $1 \Omega$ standard: typical corrections of the order $0.02 \mu \Omega / \Omega$ between $1 \mathrm{~s}$ and $340 \mathrm{~s}$ cycle times are measured. More details on these measurements can be found in [13].

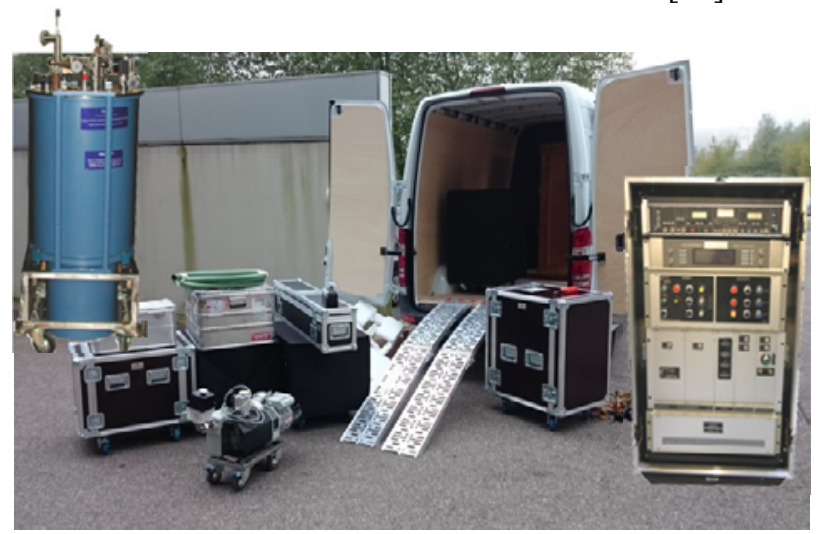

Fig. 5. View of the transportable cryostat, the bridge and the equipment used for a comparison.

\subsubsection{QHE samples}

Since the beginning of the BIPM.EM-K12 comparison program, the BIPM has used the same batch of quantum Hall samples. They are GaAs based heterostructures fabricated by the Laboratoires d'Électronique Philips (LEP, Limeil-Brévannes, 1990) [14], with a mobility $\mu$ of about $30 \mathrm{~T}^{-1}$ and a carrier concentrations $n_{c}$ of about $5.1 \times 10^{15} \mathrm{~m}^{-2}$. The samples are commonly operated on the $n=2$ plateau at a temperature around $1.3 \mathrm{~K}$, with a current of $40 \mu \mathrm{A}$ and with a magnetic flux density of about $10.5 \mathrm{~T}$. The residual values of longitudinal resistivity do not exceed $50 \mu \Omega$. A further description of the QHE device and other equipment used by the BIPM during the comparison can be found in $[9,10,11,12]$.

\section{Results}

Since the BIPM.EM-K12 comparison program resumed in 2013, four comparisons have been organized. The first one with the PTB (Germany) [9] resulted in a successful agreement between the QHE (and scaling) systems from BIPM and PTB down to the level of $1 \mathrm{n} \Omega / \Omega$ with a standard uncertainty of the order of 2 to $4 \mathrm{n} \Omega / \Omega$. This result is significantly better that could be expected from a simple comparison using classical resistance standards circulating between the two institutes [6]. This demonstrates that, although such an on-site comparison is far from being trivial to implement, this exercise is valuable to check the equivalence of NMIs at the level of 1 part in $10^{9}$.

During this first comparison, some interesting effects were found regarding the $1 \Omega$ standards [13]. It has been found that the apparent value of a $1 \Omega$ standard can vary by $0.01 \mu \Omega / \Omega$ to $0.1 \mu \Omega / \Omega$, depending on the polarity reversal cycle time of the measuring bridge used. This effect has been observed thanks to the use of a new generation of DAC-based CCC bridges allowing faster and more flexible adjustments of polarity reversal cycles compared to the analogue CCC bridges which were used during the previous series of comparisons (before 2013).

In 2015, a comparison with another NMI had to be abandoned due to technical problems with their bridge and a comparison planned in 2016 had to be postponed following unsuccessful preliminary tests showing significant discrepancies in calibration measurements performed using the NMI's QHE reference.

A comparison carried out with the CMI (Czech Republic) in 2017 led to a successful agreement, comparable to that obtain at the PTB. The comparison report is currently being written.

\begin{tabular}{|l|c|c|c|}
\hline \multicolumn{1}{|c|}{ NMI } & $\boldsymbol{x}_{\boldsymbol{i}} / \mathbf{1 0}^{-\boldsymbol{9}}$ & $\boldsymbol{u}_{\boldsymbol{i}} / \mathbf{1 0}^{-\boldsymbol{9}}$ & Date of comp. \\
LNE & -1.2 & 3.0 & Dec 1993 \\
METAS & +0.9 & 1.7 & Nov 1994 \\
PTB & -0.6 & 1.9 & Oct 1995 \\
NPL & +0.1 & 3.9 & Dec 1997 \\
NIST & +1.2 & 2.0 & Apr 1999 \\
PTB & -0.5 & 2.2 & Nov 2013 \\
CMI & To be published & - & Apr 2017 \\
\hline
\end{tabular}

Table 1. BIPM-NMI differences of ratio QHR / $100 \Omega$ for the last 25 years of BIPM.EM-K12 key comparison. Data regarding the ratios $10 \mathrm{k} \Omega / 100 \Omega$ and $100 \Omega / 1 \Omega$ can be found on the KCDB website [8].

\section{Conclusion}

The technical issues encountered during two of the comparisons since resuming the BIPM.EM-K12 program, clearly demonstrate that, although the QHE is today a well-known effect used by many NMIs to realize the ohm, its implementation at the highest accuracy level remains challenging. This confirms the importance of such comparisons and the need to increase their number as the QHE will become in the near future, after the revision of the present International System of Units [15], the natural path to realize the ohm. One can expect that further research on the QHE in graphene [16, 17] would pave the way for a simplification of the 
transportable QHE system developed at the BIPM. It has effectively been shown that the QHR can be implemented in a graphene device at liquid helium temperature $(4.2 \mathrm{~K})$ and a magnetic field of flux density of the order of 5 to $6 \mathrm{~T}$. In these conditions, a simple liquid helium reservoir and a probe including a small superconducting magnet would be sufficient, reducing significantly the number, size and weight of the equipment to transport on-site. Thus, the logistical organization, which represents an important amount of work in this kind of comparison, could be highly simplified.

However, our investigations on graphene QHE samples [18] have shown that even if good results can be obtained with such devices, their reliability is not yet sufficient for utilization in routine calibration and on-site comparisons.

Waiting for this technology to be mature, the BIPM will continue its efforts to maintain and improve its present system in order to pursue and improve the BIPM.EM-K12 program of on-site comparison.

\section{References}

1. B. Jeanneret and S.P. Benz, Eur. Phys. J. Special Topics 172, 181-206 (2009)

2. K. v Klitzing, G. Dorda, M. Pepper, Phys. Rev. Lett. 45, 494 (1980)

3. http://physics.nist.gov/cgi-bin/cuu/Value?rk90

4. F. Delahaye, IEEE Trans. Instrum. Meas., 40(6), 883-888 (1991)

5. http://www.bipm.org/fr/about-us/

6. G R Jones, B J Pritchard and R E Elmquist, Metrologia, 46, 503 (2009)

7. T. J. Quinn, "News from the BIPM", Metrologia, 26, 69-74 (1989)

8. http://kcdb.bipm.org, search for 'BIPM.EM-K12'

9. R. Goebel, N Fletcher, B Rolland, M Götz and E Pesel, Metrologia, 51, Tech. Suppl. (2014)

10. F. Delahaye, D. Bournaud, IEEE Trans. Instrum. Meas., 40(2), 237-240 (1991)

11. F. Delahaye, D. Bournaud, IEEE Trans. Instrum. Meas., 42(2), 287-291 (1993)

12. F. Delahaye, IEEE Trans. Instrum. Meas., 40(6), 883-888 (1991)

13. N. Fletcher, B Rolland, M Götz and E Pesel, , Metrologia, 52, 509 (2015)

14. F. Piquemal et al., IEEE Trans. Instrum. Meas., 42, 264-268 (1993)

15. http://www.bipm.org/en/measurement-units/rev-si/

16. W. Poirier et al, Nature Communications, 6, 6806 (2015)

17. T.J.B.M. Janssen, Reports on Progress in Physics, 76, 104501 (2013)

18. N. Fletcher, P. Gournay, B. Rolland, M. Götz, S. Novikov, N. Lebedeva, A. Satrapinski, digest of the
Conference on Precision Electromagnetic Measurements (CPEM 2016), (2016) 